\title{
An Automatic Method for Unsupervised Feature Selection of Hyperspectral Images Based on Fuzzy Clustering of Bands
}

\author{
Behnam Asghari Beirami*, Mehdi Mokhtarzade \\ Faculty of Geodesy and Geomatics, K. N. Toosi University of Technology, Tehran 19967-15433, Iran
}

Corresponding Author Email: b_asghari@email.kntu.ac.ir

https://doi.org/10.18280/ts.370218

Received: 27 December 2019

Accepted: 16 February 2020

\section{Keywords: \\ hyperspectral classification, band selection; statistical attributes, fuzzy c-means clustering, virtual dimensionality, principal component analysis}

\begin{abstract}
Hyperspectral sensors collect spectral data in numerous adjacent spectral bands which are usually redundant and cause some challenges such as Hughes phenomenon. In this study, an automatic unsupervised method is presented for feature selection from hyperspectral images. To do so, a new statistical feature space is introduced in which each band is regarded as a sample point. This feature space is originated from the statistical attributes of image bands while these attributes are extracted from different partitions of the entire image. A fuzzy clustering of bands, performed in the PCA transformed space of previously mentioned statistical feature space, leads to band clusters with similar characteristics. The proposed band selection technique chooses a representative band in each cluster and removes the other redundant ones. The proposed method is investigated in terms of the classification accuracy of the Pavia University hyperspectral image. Obtained results, which are compared to two recent states of art methods, prove its efficiency.
\end{abstract}

\section{INTRODUCTION}

Hyperspectral sensors collect data simultaneously in hundreds of narrow and adjacent spectral bands over the wavelengths from the near-ultraviolet through the thermal infrared and form a data cube that its third dimension specified by wavelengths. These sensors have great ability in the accurate reconstruction of the spectral signature curve which is a powerful tool for the identification of ground materials [1].

Redundant adjacent bands in a hyperspectral image cause storage and transmission challenges, increasing computational complexity and commonly, can decrease the performance of the classifiers [2]. Dimensionality reduction is a strategy to deal with the mentioned challenges. For hyperspectral remote sensing, dimensionality reduction references to compress the number of the bands without the decrease of the useful information [3]. Generally, dimensionality reduction methods can be categorized into two main groups; feature extraction and feature selection. The main goal of feature extraction is to find a transformation that maps data to the new low dimensional space which preserves essential discriminative information [4] but Feature selection methods attempt to select a group of bands with more information and less correlation.

Both dimensionality reduction categories again can categorize into two main subgroups: unsupervised and supervised whether it uses the labeled samples or not. In the subgroup of unsupervised feature extraction, there are wellknown methods such as Principal component analysis (PCA), independent components analysis (ICA), and minimum noise fraction (MNF) which ranks the extracted features based on some criteria [5].

There are numerous improved versions of these traditional methods in literature such as; segmented principal components analysis [6], non-linear PCA [7], Superpixel-based PCA (SuperPCA) [8], and segmented minimum noise fraction [9] for classification of hyperspectral images. In addition to these traditional approaches, there are some advanced nonlinear manifold-based methods such as; locality preserving projection (LPP) [10] and its new two stage version (TwoSP) [11] for classification of hyperspectral images. In the recent years some pixel-based methods such wavelet decomposition [12], rational curve fitting [4] and fractal dimension of spectral response curve [13] are proposed which considered the spectral curves of each pixel for extracting the new features. In the subgroup of supervised feature extraction, there are methods such as decision boundaries (DBFE) [14], nonparametric weighted feature extraction (NWFE) [15], linear discriminant analysis (LDA) [16], spectral segmentation and integration (SSI) based on PSO optimization [17].

Feature selection methods can be categorized into six subgroups as below [18]:

(1) Ranking based methods: in these methods the importance of each feature is computed and bands are sorted based on their importance and the desired number of features is selected from top-ranked ones. Information divergence [19] and mutual information [20] are the most important unsupervised and supervised methods of this subgroup.

(2) Searching-based methods: In these methods band selection problem considered as an optimization problem. Integration genetic and PSO [21] and PSO with dynamic subswarms [22] are the recent methods of this subgroup.

(3) Sparsity-based methods: In these methods band selection problem considered as a sparsity constraint optimization. sparse nonnegative matrix factorization (SNMF) [23], a collaborative sparse model [24], a group-sparsity-based algorithm [25] are well-known methods of this subgroup.

(4) Embedding learning-based method: Embedding learning-based methods select the bands for the specific application such as classification of unmixing based on optimization. modified recursive SVM (MR-SVM) belongs to 
this category [26].

(5) Clustering-based methods: in these methods, correlated bands are grouped in the same cluster and the representative band is selected from each cluster. A supervised band selection method named PSBS has been proposed by Mojaradi et al. [27] based on k-means clustering of bands. Also, its unsupervised feature extraction version is proposed by Beirami and Mokhtarzade [28].

(6) Hybrid scheme-based methods: In these methods, two or more methods of different subgroups are integrated to produce the new method. For example, clustering and ranking methods can be integrated for unsupervised band selection of the hyperspectral image [29].

Clustering-based methods are fast and efficient methods for dimensionality reduction of hyperspectral data. The idea of MartÍnez-UsÓMartinez-Uso et al. [30] is based on a hierarchical clustering structure to group bands to minimize the intra-cluster variance and maximize the inter-cluster variance. This aim is pursued using information measures, such as distances based on mutual information or KullbackLeibler divergence, in order to reduce data redundancy and non-useful information among image bands. An unsupervised method is proposed by Sohaib and Mushtaq [31] based on kmeans clustering of bands with statistical measures such as variance (Var), standard deviation (STD), and mean absolute difference (MAD). This method was later reinforced by Saqui et al. [32] who added kurtosis measure and changed the clustering method to fuzzy c-means. These researchers also introduced a new within-cluster measure for selecting the representative band of each cluster. The current study extends the 2 last aforementioned studies in different aspects as below:

(1) A comprehensive set of statistical attributes are proposed to characterize hyperspectral image bands. These attributes are used later to produce new features space for describing the bands.

(2) An image partitioning is suggested to consider the local nature of the band's statistical attributes.

To summarize the study, this paper aims to propose a new method for the unsupervised feature selection of HSI. This is done by the fuzzy clustering of HSI's bands in the rich statistical attribute state of bands. This paper consists of five sections. After the introduction, in the second section of this study, we presented band attributes and our methodology for dimensionality reduction. In the third section, we introduce the data set. Experimental results and conclusions are presented in the fourth and last section, respectively.

\section{METHODOLOGY}

As mentioned earlier, MAD and kurtosis measures are used as statistical measures [32]. It seems that using the more statistical measures can enhanced their method. As an example, consider two vectors as below:

$\mathrm{A}=[1.13,2.87,-0.38,-0.31,-0.11,1.91,1.17,-0.36,1.71$, $0.29]$

$\mathrm{B}=[1.87,2.55,-0.074,0.879,0.058,1.63,0.22,0.012,1.46$, 1.066].

Some statistical measures of these two vectors are as Table 1.

It can be understood that the largest difference between the two vectors is in iqr measure. As the results in this study, some additional statistical measures (Table 2) are considered in comparison to [32] to better describe the hyperspectral bands.
Table 1. Some statistical measures of vectors A and B

\begin{tabular}{ccc}
\hline & $\mathrm{A}$ & $\mathrm{B}$ \\
\hline Mean & 0.79 & 0.96 \\
MAD & 0.96 & 0.75 \\
Kurtosis & 1.97 & 1.86 \\
Inter quartile range (iqr) & 2.02 & 1.57 \\
\hline
\end{tabular}

Table 2. Statistical attributes of bands

\begin{tabular}{|c|c|}
\hline $\begin{array}{l}\text { Statistical } \\
\text { attributes }\end{array}$ & Formula \\
\hline $\begin{array}{l}\text { Mean absolute } \\
\text { deviation } \\
\text { (MAD) }\end{array}$ & $\mathrm{SM} 1 \mathrm{~b}=\frac{1}{M . N} \sum_{i=1}^{M . N}\left|B_{i}-\operatorname{mean}(b)\right|$ \\
\hline $\begin{array}{l}\text { Standard } \\
\text { deviation } \\
\text { (STD) }\end{array}$ & $\mathrm{SM} 2 \mathrm{~b}=\sqrt{\frac{1}{M . N} \sum_{i=1}^{M . N}\left(B_{i}-\operatorname{mean}(b)\right)^{2}}$ \\
\hline Variance (Var) & $\mathrm{SM} 2 \mathrm{~b}=\frac{1}{M N} \sum_{i=1}^{M . N}\left(B_{i}-\operatorname{mean}(b)\right)^{2}$ \\
\hline $\begin{array}{l}\text { Level-3 } \\
\text { Moment }\end{array}$ & $\mathrm{SM} 4_{\mathrm{b}}=\mathrm{E}\left(\left(B_{i}-\operatorname{mean}(b)\right)^{3}\right)$ \\
\hline Mean & $\mathrm{SM} 5 \mathrm{~b}=\frac{\sum_{i=1}^{M . N} B_{i}}{M . N}$ \\
\hline \multirow{3}{*}{ Median } & $\begin{array}{c}\text { For } B_{1}=\min , B_{2}, \ldots, B_{M . N}=\max \\
\text { If } M . N \text { is odd } \\
\text { SM } 6_{\mathrm{b}}=B_{\underline{(M . N+1)}}\end{array}$ \\
\hline & If M.N is even \\
\hline & $\begin{array}{c}\text { SM6 } \mathrm{b}=\frac{1}{2}\left(B_{\frac{(M . N)}{2}}+B_{\left.1+\frac{(M . N)}{2}\right)}\right. \\
\frac{1}{M . N} \sum_{i=1}^{M=N}\left(B_{i}-(\operatorname{mean}(b))^{4}\right.\end{array}$ \\
\hline Kurtosis & 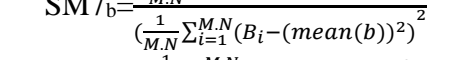 \\
\hline \multirow{2}{*}{ Skewness } & $\mathrm{SM} 8_{\mathrm{b}}=\frac{\frac{1}{M . N} \sum_{i=1}^{M . N}\left(B_{i}-(\operatorname{mean}(b))^{3}\right.}{{ }^{3}}$ \\
\hline & $\sqrt{\left(\frac{1}{M . N} \sum_{i=1}^{M . N}\left(B_{i}-(\operatorname{mean}(b))^{2}\right)^{3}\right.}$ \\
\hline $\begin{array}{l}\text { Inter quartile } \\
\text { range (irq) }\end{array}$ & $\mathrm{SM} 9_{b}=\mathrm{Q}_{3}-\mathrm{Q}_{1}$ \\
\hline
\end{tabular}

In Table 2, Bi is value of $i^{\text {th }}$ pixel, $M$ and $N$ show image dimensions, mean $(b)$ is mean of all pixel values in band $b$ and Q3, $Q 1$ are third and first quartile. Figure 1 shows the flowchart of the proposed method. The details are presented below:

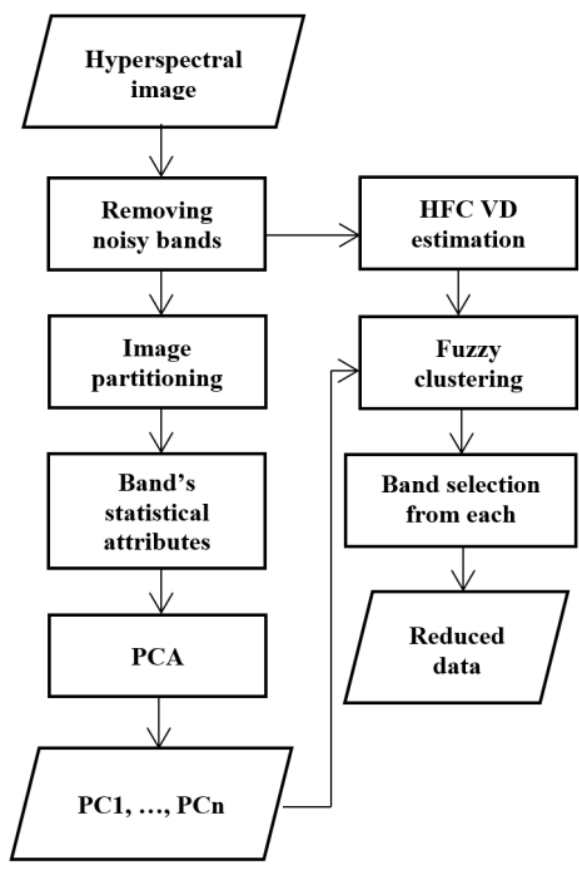

Figure 1. Flowchart of proposed method 
Noisy bands (such as water vapor bands) have no useful information and thus the proposed method begins by removing them manually via visual inspection. The remained bands are then introduced to Harsanyi, Farrand, and Chang (HFC), wellknown virtual dimensionality (VD) estimation algorithm [33], to estimate the number of distinct signatures.

Image-space is spatially partitioned to equal size segments (Figure 2). This is due to the elongation of the image object illustrated along the width of the image (such as road and buildings). An attribute feature space is then formed by extracting some statistical attributes from each image segment and for each hyperspectral band. It can help our method catches the local characteristics of each band in comparison to other previous methods.

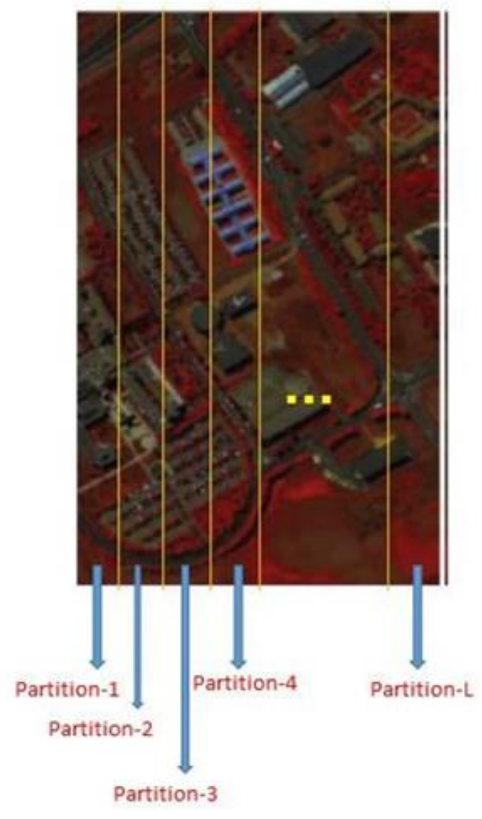

Figure 2. Image partitioning

These nine features, if extracted from L image partition, lead to $9 \times \mathrm{L}$ attributes for each band which means that bands can be regarded as points in that $9 \times \mathrm{L}$ dimension space. In other words, band $\mathrm{b}$ can be described by a vector in the form of Eq. (1):

$$
\boldsymbol{b}=[S M 11, \ldots, S M 91, \ldots, S M 1 L, \ldots, S M 1 L]^{\mathrm{T}}
$$

where, $S M 11, \ldots, S M 91$ represent the nine statistical attributes of Table 2 and $\mathrm{L}$ is the number of image partitions. The components of Eq. (1) are the statistical attributes of the bands. In the next stage, a PCA was applied to band attributes space for extracting the most useful attributes of each band. PCA is the linear transformation that aims to find transformation matrix $W$ that transform the original features space $b$ to the new uncorrelated feature space $y$ as below:

$$
y_{i}=W^{T} b_{i}
$$

Each hyperspectral band can be represented by a $9 \times \mathrm{L}$ directional vector as $b=\left[S M_{1}, S M_{2}, \ldots, S M_{9 \times L}\right]$. If hyperspectral image contains the $M$ bands the covariance matrix of $x$ is computed as [34]:

$$
\operatorname{cov}(b)=E\left\{\left(b-E(b)\left(b-E(b)^{T}\right)\right\}\right.
$$

where, $E$ is expectation operator and $T$ is the transpose. The covariance matrix can be represented in eigenvalue decomposition as below:

$$
\operatorname{cov}(x)=W D W^{T}
$$

where, $D$ is the diagonal matrix which consists of eigenvalues of $\operatorname{cov}(x)$ and $W$ is the orthogonal matrix of eigenvectors of $\operatorname{cov}(x)$. Commonly, eigenvectors correspond to the $k$ largest eigenvalues are used in (2) as the transformation matrix [34].

As the same motivation which is proposed by Saqui at al. [32], Fuzzy c-means clustering was applied to cluster the bands in the principal components space for VD number of clusters. Fuzzy c-means clustering aims to minimize of the following objective function [35]:

$$
J_{m}=\sum_{i=1}^{M} \sum_{j=1}^{V D} u_{i j}^{m}\left\|b_{i}-c_{j}\right\|^{2} \quad 1 \leq m \leq \infty
$$

where, $u_{i j}$ is the degree of membership of $b_{i}$ in the cluster $j, c_{j}$ center of the $j^{\text {th }}$ cluster. An iterative method is carried out to solve the above optimization problem with update of membership and cluster center as below [35]:

$$
\begin{gathered}
u_{i, j}=\frac{1}{\sum_{k=1}^{V D}\left(\frac{\left\|b_{i}-c_{j}\right\|}{\left\|b_{i}-c_{k}\right\|}\right)^{\frac{2}{m-1}}} \\
c_{j}=\frac{\sum_{i=1}^{M} u_{i j}^{m} \cdot b_{i}}{\sum_{i=1}^{M} u_{i j}^{m}}
\end{gathered}
$$

The iterative procedure will stop when the changes in the value of $u_{i, j}$ is small. It is assumed that the bands with similar information content would appear to have high membership values to common clusters. Accordingly, a band with a maximum degree of membership was selected as the representative band of each cluster and the other bands were removed as they were thought to be redundant.

\section{DATA SET}

The data set of this study is acquired by the ROSIS-3 sensor from Pavia University from in northern Italy on 8 July 2002 and has a size of $610 \times 340$ pixels with $1.3 \mathrm{~m}$ spatial resolution. Each image pixel contains 115 reflectance values from 0.43 to 0.86 micrometers of the spectrum. 12 noisy bands were removed and the remaining 103 bands were used in this study. Pavia University's three-channel color composite and groundtruth map are shown in Figure 3. In addition, Table 3 shown the total numbers of samples in each class based on ground truth.

Table 3. Ground truth samples distribution

\begin{tabular}{cc}
\hline Class & No. of sample in ground truth \\
\hline asphalt & 6631 \\
Meadows & 18649 \\
Gravel & 2099 \\
Trees & 3064 \\
Painted metal sheets & 1345 \\
Bare Soil & 5029 \\
Bitumen & 1330 \\
Self-Blocking Bricks & 3682 \\
Shadows & 947 \\
Total & $\mathbf{4 2 7 7 6}$ \\
\hline
\end{tabular}



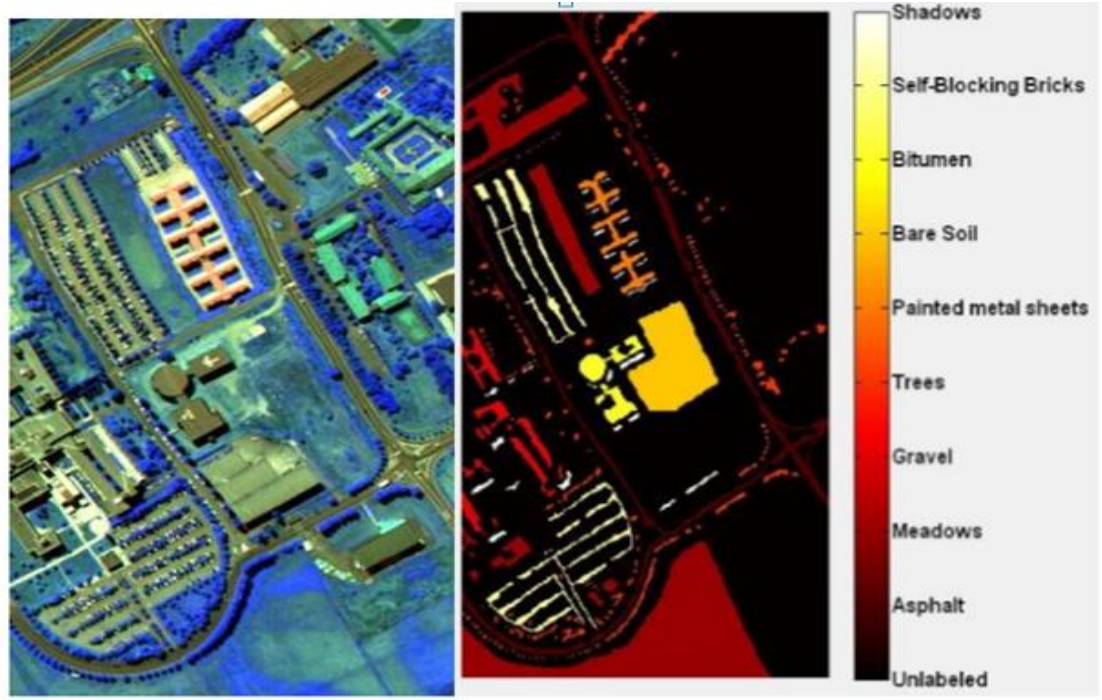

(a) Color composite image (b) Ground truth map

Figure 3. Pavia University

\section{EXPERIMENTAL RESULTS}

The VD of Pavia University data set was estimated by HFC based on $10-5$ as a false alarm which found to be 13 dimensions. This VD number determines the number of clusters in the latter fuzzy c-means clustering. Due to the randomness of the fuzzy c-mean clustering initialization, clustering was performed ten times and the one with the lowest clustering objective function [35] was chosen. The proposed band selection method was evaluated in terms of classification accuracy based on overall accuracy (OA) and kappa coefficient (Kappa) measures as below:

$$
\begin{gathered}
\mathrm{OA}=\frac{a}{A} \\
\text { kappa }=\frac{A \sum_{i=1}^{r} X_{i i}-\sum_{i=1}^{r} X_{i+} X_{+i}}{A^{2}-\sum_{i=1}^{r} X_{i+} X_{+i}}
\end{gathered}
$$

where, in 8, $A$ and $a$ are the total number of test and correctly classified samples, respectively. In (9), $X_{i i}$ is the total number of samples belonging to the class $i$ that have also been classified as class $i$ (diagonal elements of the confusion matrix). $X_{i+}$ and $X_{+i}$ are the sum of all columns and all rows of error matrix for class $i$. Also, $r$ is the total number of classes in ground truth.

For the sake of the classification, selected bands (features) are classified based on K-nearest neighbor (K-NN) classification. K-nearest neighbor $(\mathrm{K}-\mathrm{NN})$ algorithm is the non-parametric supervised simple classification method that has the appropriate results in the classification of hyperspectral images. In this method the label of each test sample is determined based on the K-nearest labeled sample. Commonly Euclidean metric $(E M)$ is used to measure the distance between each test $(x)$ and labeled $(y)$ sample as below [36]:

$$
E M(x, y)=\sqrt{\sum_{i=1}^{n}\left|x_{i}-y_{i}\right|^{2}}
$$

where, $n$ is the number of the features. Training samples were randomly selected as the $10 \%$ of ground truth samples and the remaining ones were applied to evaluate the classification results.

\subsection{Effects of PCs}

In the first experiment, image partitioning was not performed and only nine statistical attributes were calculated for each band of hyperspectral image. PCA was applied to these band statistical attributes and led to new uncorrelated principal components (PCs). Different numbers of PCs, from 1 to 4 , were used in the proposed band selection to examine the effects of this parameter. Reduced data of each case was evaluated using overall classification accuracy (Figure 4).

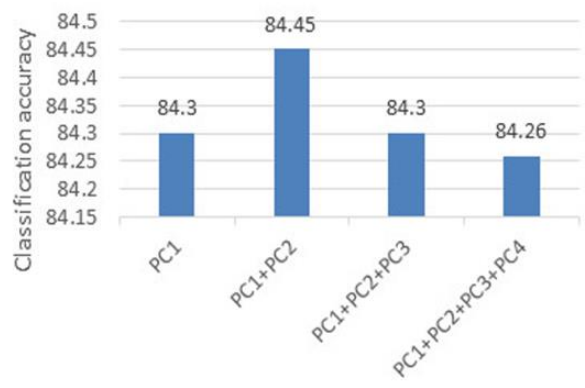

Figure 4. Effect of different number of PCs

\subsection{Effects of image partitioning}

The second experiment aims to evaluate the effects of image partitioning on the performance of the proposed method. To do so, different numbers of image partitions (i.e. 1 to 8 ) were evaluated. The obtained results are presented in Figure 5.

Figure 5 suggests that image partitioning has significant effects on the performance of the proposed method. This means that statistical attributes associated to each band have local nature. The optimum number of image partitions is 6 in our case. This type of partitioning is used due to its simplicity and very fast nature. But more precisely, this parameter, as well as the proper method of image partitioning, needs to be the subject of more research in future. According to Figure 5, even with two image partitions classification accuracy of selected bands exceed the minimum mapping accuracy of $85 \%$ which is required for most resource management applications [37]. 


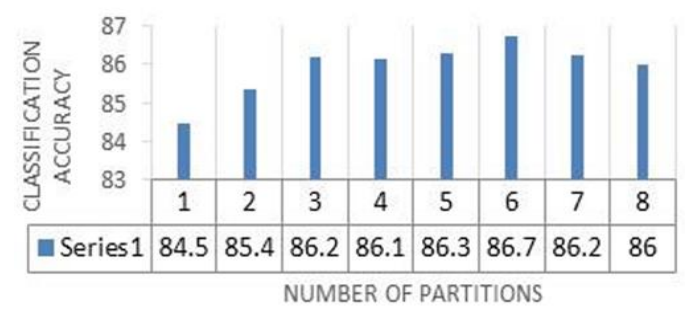

Figure 5. Effect of image partisioning

\subsection{Comparison to other methods}

The proposed method was compared to the studies [31] and [32] which are the most recent unsupervised band selection techniques with a similar concept to the proposed method. Figure 6 shows the obtained results which indicate the superiority of the proposed method. Both of the two opponent methods, as well as the proposed method, introduce their selected bands based on band clustering. The superiority of the proposed method can be traced to two factors:

(1) The rich original statistical attributes which were assigned to each band (Table 2).

(2) The strategy of image partitioning which describes the bands more efficiently.

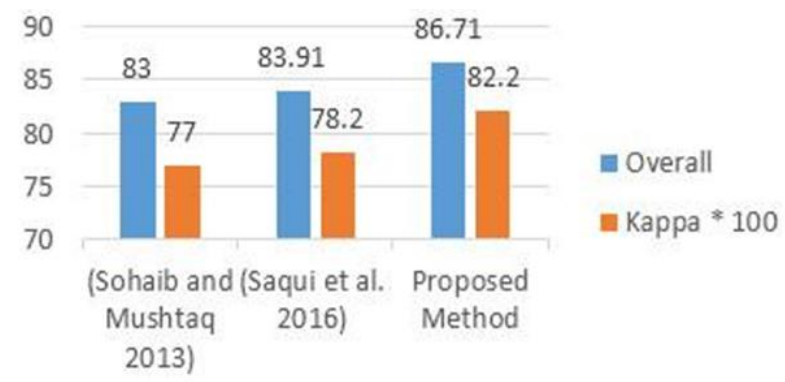

Figure 6. Results of different methods

In comparison to classification with original spectral bands (overall $=86.16$ ), only our proposed method is /superior. This indicates that our method can efficiently reduce the dimension of data to about 0.14 along with the preservation of important original data. Besides, although the proposed method is feature selection, it is still more precise than the PCA feature extraction method (13 first PCs) with an overall accuracy of $86.15 \%$.

\section{CONCLUSION}

In this paper, an automatic unsupervised band selection technique was proposed for the sake of dimensionality reduction of hyperspectral images. The underlying concept of the proposed method is assigning some statistical attributes to each band and then clustering them accordingly. A representative band in each cluster was kept and the remaining ones were assumed to be redundant. To examine the performance of the proposed method, the resultant bands were used in K-NN classification and the results were evaluated in terms of overall accuracy and kappa coefficients for the test samples. The accuracy results of the proposed method are higher than the other two recent methods of this field and the PCA feature extraction method when the same set of training and test samples is chosen which proved the efficiency of the proposed method.

The band selection method of this paper applied an image partition that has a significant impact on its functionality. Determination of the proper number and strategy of this partitioning is proposed for further study.

\section{REFERENCES}

[1] Wei, Y., Zhu, X., Li, C., Guo, X., Yu, X., Chang, C., Sun, H. (2017). Applications of hyperspectral remote sensing in ground object identification and classification. Advances in Remote Sensing, 6(3): 201-211. https://doi.org/10.4236/ars.2017.63015

[2] Hughes, G. (1968). On the mean accuracy of statistical pattern recognizers. IEEE Transactions on Information Theory, 14(1): 55-63. https://doi.org/10.1109/TIT.1968.1054102

[3] Su, H., Sheng, Y., Du, P. (2008). A new band selection algorithm for hyperspectral data based on fractal dimension. The International Archives of the Photogrammetry, Remote Sensing and Spatial Information Sciences, 37(B7): 279-284.

[4] Hosseini, S.A., Ghassemian, H. (2016). Rational function approximation for feature reduction in hyperspectral data. Remote Sensing Letters, 7(2): 101110. https://doi.org/10.1080/2150704X.2015.1101180

[5] Chang, C.I. (2013). Hyperspectral data processing: algorithm design and analysis. John Wiley \& Sons.

[6] Jia, X., Richards, J.A. (1999). Segmented principal components transformation for efficient hyperspectral remote-sensing image display and classification. IEEE transactions on Geoscience and Remote Sensing, 37(1): 538-542. https://doi.org/ 10.1109/36.739109

[7] Licciardi, G., Chanussot, J. (2018). Spectral transformation based on nonlinear principal component analysis for dimensionality reduction of hyperspectral images. European Journal of Remote Sensing, 51(1): 375-390. https://doi.org/10.1080/22797254.2018.1441670

[8] Jiang, J., Ma, J., Chen, C., Wang, Z., Cai, Z., Wang, L. (2018). SuperPCA: A superpixelwise PCA approach for unsupervised feature extraction of hyperspectral imagery. IEEE Transactions on Geoscience and Remote Sensing, 56(8): 4581-4593. https://doi.org/10.1109/TGRS.2018.2828029

[9] Guo, L.X., Xie, W.X., Pei, J.H. (2015). Segmented minimum noise fraction transformation for efficient feature extraction of hyperspectral images. Pattern Recognition, 48(10): 3216-3226. https://doi.org/10.1016/j.patcog.2015.04.013

[10] He, X., Niyogi, P. (2004). Locality preserving projections. In Advances in Neural Information Processing Systems, pp. 153-160.

[11] Li, X., Zhang, L., You, J. (2018). Hyperspectral image classification based on two-stage subspace projection. Remote $\quad$ Sensing, $10(10)$ : 1565. https://doi.org/10.3390/rs10101565

[12] Prabhu, N., Arora, M.K., Balasubramanian, R. (2016). Wavelet based feature extraction techniques of hyperspectral data. Journal of the Indian Society of Remote Sensing, 44(3): 373-384. https://doi.org/10.1007/s12524-015-0506-9 
[13] Ghosh, J.K., Somvanshi, A. (2008). Fractal-based dimensionality reduction of hyperspectral images. Journal of the Indian Society of Remote Sensing, 36(3): 235-241. https://doi.org/10.1007/s12524-0080024-0

[14] Lee, C., Landgrebe, D.A. (1993). Feature extraction based on decision boundaries. IEEE Transactions on Pattern Analysis and Machine Intelligence, 15(4): 388400. https://doi.org/ 10.1109/34.206958

[15] Kuo, B.C., Landgrebe, D.A. (2004). Nonparametric weighted feature extraction for classification. IEEE Transactions on Geoscience and Remote Sensing, 42(5): 1096-1105. https://doi.org/ 10.1109/TGRS.2004.825578

[16] David, L. (2002). Hyperspectral image data analysis as a high dimensional signal processing problem. IEEE Signal Processing Mag, 19(1): 17-28.

[17] Moghaddam, S.H.A., Mokhtarzade, M., Beirami, B.A. (2020). A feature extraction method based on spectral segmentation and integration of hyperspectral images. International Journal of Applied Earth Observation and Geoinformation, $\quad 89$ 102097 https://doi.org/10.1016/j.jag.2020.102097

[18] Sun, W., Du, Q. (2019). Hyperspectral band selection: A review. IEEE Geoscience and Remote Sensing Magazine, $7(2)$ :

118-139. https://doi.org/10.1109/MGRS.2019.2911100

[19] Chang, C.I., Liu, K.H. (2013). Progressive band selection of spectral unmixing for hyperspectral imagery. IEEE Transactions on Geoscience and Remote Sensing, 52(4): 2002-2017. https://doi.org/10.1109/TGRS.2013.2257604

[20] Guo, B., Gunn, S.R., Damper, R.I., Nelson, J.D. (2006). Band selection for hyperspectral image classification using mutual information. IEEE Geoscience and Remote Sensing Letters, 3(4): 522-526. https://doi.org/10.1109/LGRS.2006.878240

[21] Ghamisi, P., Benediktsson, J.A. (2014). Feature selection of hyperspectral data by considering the integration of genetic algorithms and particle swarm optimization. In Image and Signal Processing for Remote Sensing XX, 9244: 92440J. https://doi.org/10.1117/12.2065472

[22] Xu, M., Shi, J., Chen, W., Shen, J., Gao, H., Zhao, J., (2018). A band selection method for hyperspectral image based on particle swarm optimization algorithm with dynamic sub-swarms. Journal of Signal Processing Systems, $\quad 90(8-9)$ :

1269-1279. https://doi.org/10.1007/s11265-018-1348-9

[23] Li, J.M., Qian, Y.T. (2011). Clustering-based hyperspectral band selection using sparse nonnegative matrix factorization. Journal of Zhejiang University $\begin{array}{llll}\text { Science } & \text { C, } & \text { 12(7): }\end{array}$ https://doi.org/10.1631/jzus.C1000304

[24] Du, Q., Bioucas-Dias, J.M., Plaza, A. (2012). Hyperspectral band selection using a collaborative sparse model. In IEEE International Geoscience and Remote Sensing Symposium, pp. 3054-3057. https://doi.org/10.1109/IGARSS.2012.6350781

[25] Li, H., Wang, Y., Duan, J., Xiang, S., Pan, C. (2013). Group sparsity based semi-supervised band selection for hyperspectral images. In IEEE International Conference on Image Processing, pp. 3225-3229. https://doi.org/10.1109/ICIP.2013.6738664
[26] Zhang, R., Ma, J., Chen, X., Tong, Q. (2009). Feature selection for hyperspectral data based on modified recursive support vector machines. In IEEE International Geoscience and Remote Sensing Symposium, 2: II-847. https://doi.org/10.1109/IGARSS.2009.5418228

[27] Mojaradi, B., Emami, H., Varshosaz, M., Jamali, S. (2008). A novel band selection method for hyperspectral data analysis. The International Archives of the Photogrammetry, Remote Sensing and Spatial Information Sciences, Beijing, China, pp. 447-454.

[28] Beirami, B.A., Mokhtarzade, M. (2019). Introducing an unsupervised method for feature extraction of hyperspectral images using bands clustering in the prototype space. Journal of Geomatics Science and Technology, 9(2): 195-207.

[29] Datta, A., Ghosh, S., Ghosh, A. (2015). Combination of clustering and ranking techniques for unsupervised band selection of hyperspectral images. IEEE Journal of Selected Topics in Applied Earth Observations and Remote Sensing, 8(6): 2814-2823. https://doi.org/10.1109/JSTARS.2015.2428276

[30] MartÍnez-UsÓMartinez-Uso, A., Pla, F., Sotoca, J. M., García-Sevilla, P. (2007). Clustering-based hyperspectral band selection using information measures. IEEE Transactions on Geoscience and Remote Sensing, 45(12): $4158-4171$ https://doi.org/10.1109/TGRS.2007.904951

[31] Sohaib, M., Mushtaq, Q. (2013). Dimensional reduction of hyperspectral image data using band clustering and selection based on statistical characteristics of band images. International Journal of Computer and $\begin{array}{lll}\text { Communication } & \text { Engineering, } 2(2): & 101 .\end{array}$ https://doi.org/10.7763/IJCCE.2013.V2.148

[32] Saqui, D., Saito, J.H., Campos, J.R., Jorge, L.A.D.C. (2016). Approach based on fuzzy c-means for band selection in hyperspectral images. International Journal of Computer and Information Engineering, 10(5): 889895. https://doi.org/10.5281/zenodo.1124265

[33] Harsanyi, J.C., Farrand, W.H., Chang, C.I. (1993). Determining the number and identity of spectral endmembers: An integrated approach using NeymanPearson eigen-thresholding and iterative constrained RMS error minimization. In Proceedings of the Thematic Conference on Geologic Remote Sensing, pp. 395-395.

[34] Rodarmel, C., Shan, J. (2002). Principal component analysis for hyperspectral image classification. Surveying and Land Information Science, 62(2): 115122.

[35] Bezdek, J. C., Ehrlich, R., Full, W. (1984). FCM: The fuzzy c-means clustering algorithm. Computers \& Geosciences, 10(2-3): 191-203. https://doi.org/10.1016/0098-3004 (84)90020-7

[36] Prasatha, V.S., Alfeilate, H.A.A., Hassanate, A.B., Lasassmehe, O., Tarawnehf, A.S., Alhasanatg, M.B., Salmane, H.S.E. (2017). Effects of distance measure choice on KNN classifier performance-a review. arXiv preprint arXiv:1708.04321.

[37] Townshend, J.R., Hardy, J.R., Justice, C.O., Williams, D.F., Mitchell, C.W., Cook, A., Hancock, P. (1981). Terrain Analysis and Remote Sensing. London: George Allen \& Unwin. 